\title{
Identifying and treating codeine dependence: a systematic review
}

\author{
Suzanne Nielsen', Tim MacDonald ${ }^{2,3}$, Jacinta L Johnson ${ }^{4,5}$
}

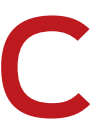

odeine is globally the most frequently used opiate, ${ }^{1}$ and its consumption is increasing. In Australia, 27780234 packs of codeine-containing analgesics were supplied by community pharmacies during 2013, a rate of 1.24 packs per person. $^{2}$ In New Zealand, most of Canada, South Africa, Ireland, and the United Kingdom, codeine is available over the counter, usually combined with simple analgesics such as paracetamol or ibuprofen; ${ }^{3}$ until recently, it was also available without prescription in Australia and France. Products containing greater amounts of codeine are generally only available on prescription. ${ }^{3}$

Codeine has low affinity for and intrinsic activity at $\mu$-opioid receptors, and is considered a prodrug; its analgesic effects depend largely on its being converted to morphine by the polymorphic cytochrome P450 isoenzyme (CYP) 2D6. ${ }^{4,5}$ Genetic variability in the activity of CYP2D6 underlie interperson differences in the analgesia achieved and the risk of opioid toxicity. ${ }^{6}$ Tolerance can develop after a relatively short period of regular use. ${ }^{7-9}$

In view of the limited evidence that adding low dose codeine $(<30 \mathrm{mg})$ to simple analgesics increases pain relief, ${ }^{10-15}$ the variability in its metabolism, and the availability of opioids with more predictable effects, the role of codeine in pain management is contentious. ${ }^{16,17}$

The liability of codeine to be misused has been shown in a randomised, double blind, placebo-controlled drug administration study, ${ }^{18}$ and has been documented in several case series. $^{19,20}$ Although the prevalence of codeine dependence is unknown, the harms associated with overuse are well established, including serious morbidity causing great cost to the health care system. ${ }^{21}$

Some harms associated with codeine overuse are directly related to prolonged intake, but many serious consequences stem from concomitant overconsumption of ibuprofen or paracetamol in combination products. ${ }^{19}$ Sequelae of supratherapeutic ibuprofen ingestion secondary to codeine dependence that require intensive care have been described, including several codeine-related deaths. ${ }^{22}$ As a result, access to over-the-counter codeine has been restricted or removed in Manitoba (February 2016), France (July 2017), and Australia (February 2018). ${ }^{23-25}$

In order to respond appropriately, we need to identify people who are codeine-dependent. There has been greater awareness of dependence with the imminent rescheduling of codeine in Australia. Both in Australia and internationally, presentations to addiction treatment services have increased, ${ }^{26-28}$ but treatment approaches for codeine dependence are poorly defined. The purpose of our systematic review was to identify the characteristics of people who are codeinedependent, and to define approaches for identifying codeine dependence.

\section{Abstract}

Objectives: Codeine dependence is a significant public health problem, motivating the recent rescheduling of codeine in Australia (1 February 2018). To provide information for informing clinical responses, we undertook a systematic review of what is known about identifying and treating codeine dependence.

Study design: Articles published in English that described people who were codeine-dependent or a clinical approach to treating people who were codeine-dependent, without restriction on year of publication, were reviewed. Articles not including empirical data were excluded. One researcher screened each abstract; two researchers independently reviewed full text articles. Study quality was assessed, and data were extracted with standardised tools.

Data sources: MEDLINE and EMBASE were searched for relevant publications on 22 November 2016. The reference lists of eligible studies were searched to identify further relevant publications. 2150 articles were initially identified, of which 41 were eligible for inclusion in our analysis.

Data synthesis: Studies consistently reported specific characteristics associated with codeine dependence, including mental health comorbidity and escalation of codeine use attributed to psychiatric problems. Case reports and series described codeine dependence masked by complications associated with overusing simple analgesics and delayed detection. Ten studies described the treatment of codeine dependence. Three reports identified a role for behavioural therapy; the efficacy of CYP inhibitors in a small open label trial was not confirmed in a randomised controlled trial; four case series/chart reviews described opioid agonist therapy and medicated inpatient withdrawal; two qualitative studies identified barriers related to perceptions of codeine-dependent people and treatment providers, and confirmed positive perceptions and treatment outcomes achieved with opioid agonist treatments.

Conclusion: Strategies for identifying problematic codeine use are needed. Identifying codeine dependence in clinical settings is often delayed, contributing to serious morbidity. Commonly described approaches for managing codeine dependence include opioid taper, opioid agonist treatment, and psychological therapies. These approaches are consistent with published evidence for pharmaceutical opioid dependence treatment and with broader frameworks for treating opioid dependence.

PROSPERO registration: CRD42016052129.

\section{Methods}

\section{Search strategy}

We searched MEDLINE and EMBASE on 22 November 2016 for the following terms: "codeine", "dependence", "substance-related disorders", "opioid-related disorders", "behaviour, addictive", and "substance withdrawal syndrome" (online Appendix, table 1). We restricted our search to human studies published in English; 
there was no restriction on year of publication. The reference lists of eligible studies were searched to identify further relevant publications.

One reviewer ( $\mathrm{SN}, \mathrm{JJ}$ or $\mathrm{TM}$ ) independently examined the titles and abstracts of identified articles. The full text of relevant articles was independently assessed for inclusion by two authors, and reasons for exclusion documented as appropriate. Inter-reviewer disagreement about inclusion was resolved by consensus among all three authors.

\section{Study inclusion criteria}

We included studies that described people who were codeinedependent (identification studies) or any clinical approach for treating people who were codeine-dependent (treatment studies).

Data extracted from identification studies included study characteristics (author, location, design, quality rating) and population characteristics (participant age, sex, employment, mental health, pain and substance use history, adverse effects related to codeine use, and management of adverse effects).

Treatment studies included randomised and non-randomised controlled trials, quasi-experimental, before-and-after studies, prospective and retrospective cohort studies, case-control studies, analytic cross-sectional studies, qualitative studies, and case reports and series. Treatment outcome measures included change in codeine use (days of use or amount used), retention in treatment, adverse events and other outcomes related to codeine use, opioid dependence, and pain.

\section{Exclusion criteria}

Reports limited to describing the clinical applications or pharmacology of codeine or other opioids, reports that did not separately report codeine-related data, and articles without empirical data (eg, letters, commentaries, reviews) were excluded (online Appendix, table 2).

\section{Assessment of methodological quality (identification studies)}

The quality of descriptive studies was assessed with a modified version of the Joanna Briggs Institute (JBI) Critical Appraisal Checklist for Analytical Cross Sectional Studies. ${ }^{29}$ To enable application of a single tool to all study methodologies, the JBI tool was reduced from eight to five items, and an item from the Evidence-Based Librarianship (EBL) Critical Appraisal Tool for assessing sample bias was added ${ }^{30}$ (online Appendix, table 3 ). The range for total scores was $0-6$, higher scores reflecting higher quality.

\section{Grading of evidence (treatment studies)}

Studies examining treatment approaches were scored for quality according to GRADE criteria. ${ }^{31}$

\section{Data collection}

Data were extracted with a standardised data extraction tool into an Excel (Microsoft) spreadsheet. The tool was piloted and reviewed before being finalised.

\section{Data synthesis}

Findings were qualitatively and quantitatively synthesised when population characteristics were reported in a manner that enabled this approach. Meta-analysis of treatment studies was not possible because of the heterogeneity of study designs. When individual patient data were reported, details were extracted at the patient level to enable synthesis of patient characteristics.

\section{Results}

Of the 2150 articles initially identified, 41 were eligible for inclusion in our analysis (Box 1). The mean study quality score of the included articles was 3.0 (standard deviation [SD], 1.1).

\section{Identifying codeine dependence}

Fourteen reports described samples of patients who were codeine-dependent (Box 2; online Appendix, table 4A); 22 described presentations by individual patients (Box 3, Box 4; online Appendix, tables $4 \mathrm{~B}$ and $\mathrm{C}$ ). No studies reported developing an approach for identifying people with codeine dependence as an aim, but two reported applying the Severity of Dependence Scale $(\mathrm{SDS})^{32}$ for defining codeine dependence (cut-off score, 5). ${ }^{33,34}$

\section{Analyses of administrative data}

Three studies examined data from administrative sources on the treatment of people for opioid dependence. ${ }^{28,35,36}$ An Australian study compared codeine-related treatment episodes with those for patients for whom another prescribed opioid or heroin was the chief drug of concern. The proportion of women among those treated for codeine dependence declined from $70 \%$ in 2002 to $47 \%$ in 2011; people for whom codeine was the drug of concern were on average older and less likely to have a history of intravenous and illicit substance use than those treated for misuse of stronger prescription opioids or heroin. ${ }^{28}$ A study of codeine prescriptions in Norway found that $0.5 \%$ of all codeine recipients in 2005 were likely to be using codeine problematically (annual prescription level exceeding twice the maximum daily dose for

1 Preferred Reporting Items for Systematic Reviews and Meta-Analyses (PRISMA) diagram of study selection

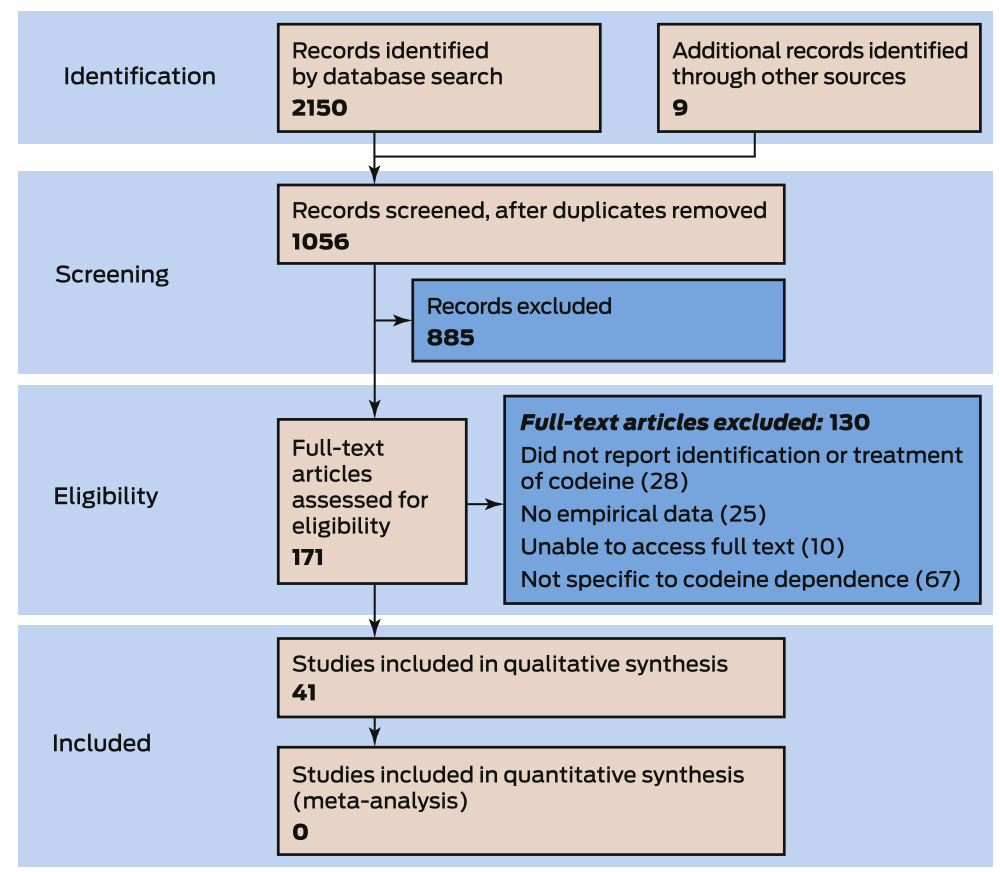


2 Studies describing characteristics of people who were codeine-dependent

\begin{tabular}{|c|c|c|c|c|c|}
\hline Study & Design, location & Sample size, type & $\begin{array}{c}\text { Sex } \\
\text { (women) }\end{array}$ & $\begin{array}{c}\text { Age } \\
\text { (years) }\end{array}$ & $\begin{array}{l}\text { Quality } \\
\text { rating }\end{array}$ \\
\hline Sproule $1999^{20}$ & Survey, Canada & $\begin{array}{l}339 \text { people who had used codeine } \\
3 \text { days/week for at least } 6 \text { months }\end{array}$ & $51 \%$ & $\begin{array}{l}\text { mean, } 43.5 \\
\text { (range, 18-82) }\end{array}$ & 5 \\
\hline Fredhein $2009^{35}$ & $\begin{array}{l}\text { National prescription database, } \\
\text { Norway }\end{array}$ & $\begin{array}{l}385190 \text { people prescribed codeine } \\
\text { during } 2005\end{array}$ & $56 \%$ & $\begin{array}{l}\text { mean, } 52.3 \\
(\mathrm{SD}, 18.8)\end{array}$ & 5 \\
\hline Frei $2010^{19}$ & $\begin{array}{l}\text { Case series of patients who } \\
\text { presented or were referred to } \\
\text { hospital addiction medicine, } \\
\text { Australia }\end{array}$ & $\begin{array}{l}27 \text { treatment admissions for serious harms } \\
\text { from over-the-counter ibuprofen-codeine } \\
\text { products }\end{array}$ & $48 \%$ & $\geq 20$ & 3 \\
\hline Thekiso $2010^{42}$ & $\begin{array}{l}\text { Retrospective chart review, } \\
\text { Ireland* }\end{array}$ & $\begin{array}{l}20 \text { inpatients admitted with over-the- } \\
\text { counter codeine abuse or dependence }\end{array}$ & $65 \%$ & $\begin{array}{l}\text { mean, } 49 \\
(\mathrm{SD}, 23)\end{array}$ & 4 \\
\hline McAvoy $2011^{40}$ & Case series, New Zealand* & $\begin{array}{l}15 \text { patients presenting for over-the-counter } \\
\text { codeine detoxification }\end{array}$ & $46 \%$ & $\begin{array}{c}\text { mean, } 44 \\
\text { (range, } 30-60 \text { ) }\end{array}$ & 3 \\
\hline McDonough $2011^{41}$ & $\begin{array}{l}\text { Retrospective chart review, } \\
\text { Victoria }\end{array}$ & $\begin{array}{l}32 \text { people referred to hospital drug and } \\
\text { alcohol services for excessive over-the- } \\
\text { counter codeine use (Sept } 2005 \text { - } \\
\text { Sept } 2010 \text { ) }\end{array}$ & $72 \%$ & median, 38 & 2 \\
\hline Nielsen $2011^{33}$ & Online survey, Australia & $\begin{array}{l}137 \text { people who met criteria for codeine } \\
\text { dependence (subset of } 800 \text { people } \\
\text { reporting over-the-counter codeine use) }\end{array}$ & $66 \%$ & $\begin{array}{l}\text { mean, } 37 \\
(S D, 13)\end{array}$ & 3 \\
\hline Cooper $2013^{38}$ & $\begin{array}{l}\text { Qualitative interviews, United } \\
\text { Kingdom* }\end{array}$ & $\begin{array}{l}25 \text { participants recruited from internet } \\
\text { support groups }\end{array}$ & $52 \%$ & $20-60 s$ & 3 \\
\hline Nielsen $2013^{39}$ & $\begin{array}{l}\text { Survey (qualitative research } \\
\text { methodology), Australia }\end{array}$ & 20 codeine-dependent people & $60 \%$ & $\begin{array}{l}\text { mean, } 39 \\
(\mathrm{SD}, 11)\end{array}$ & 2 \\
\hline Dada $2015^{36}$ & $\begin{array}{l}\text { National administrative treatment } \\
\text { dataset, South Africa }\end{array}$ & $\begin{array}{l}425 \text { codeine-related admissions to } \\
\text { alcohol and other drug treatment } \\
\text { centres, } 137 \text { with codeine as drug } \\
\text { of primary concern }\end{array}$ & $25 \%$ & $\begin{array}{c}11-70 \\
\text { (20-39 years: 59\%) }\end{array}$ & 2 \\
\hline Nielsen $2015^{28}$ & $\begin{array}{l}\text { Cross-sectional study of national } \\
\text { administrative datasets, Australia }\end{array}$ & 4424 codeine treatment episodes & $\begin{array}{l}70 \%(2002) \\
47 \%(2011)\end{array}$ & mean, 36 & 2 \\
\hline Nielsen $2015^{27}$ & $\begin{array}{l}\text { Retrospective chart review, } \\
\text { Australia* }\end{array}$ & 135 (53 codeine-dependent) & $66 \%$ & $\begin{array}{l}\text { mean, } 38 \\
(\mathrm{SD}, 8)\end{array}$ & 4 \\
\hline Qiu $2015^{37}$ & $\begin{array}{l}\text { Case-control study mapping } \\
\text { brain differences, China }\end{array}$ & $\begin{array}{l}60 \text { ( } 30 \text { codeine-dependent people, } 30 \\
\text { controls) }\end{array}$ & $7 \%$ & mean, 25 & 2 \\
\hline Van Hout $2015^{34}$ & Qualitative interviews, Ireland* & $\begin{array}{l}21 \text { participants recruited through drug } \\
\text { treatment centres (in treatment or in } \\
\text { recovery for over-the-counter codeine use) }\end{array}$ & $57 \%$ & $\begin{array}{l}\text { mean, } 39 \\
\text { (range, 26-62) }\end{array}$ & 2 \\
\hline
\end{tabular}

$\mathrm{SD}=$ standard deviation. * Study also included in Box 5 (treatment approaches). For further details, see online Appendix, table 4A.

12 months); further, high dose prescribing of benzodiazepines was more prevalent in this group. ${ }^{35}$ A South African study of national data on treatment for substance misuse (alcohol, pharmaceutical and illicit drugs) found that $2.5 \%$ of admissions involved codeine, and that codeine was recorded as the primary substance of concern for $0.8 \%$ of patients. $^{36}$

\section{Quantitative convenience samples}

Two studies included convenience samples of people who reported using codeine. In an Australian web-based survey, 137 codeine-dependent participants were compared with 633 nondependent participants; characteristics associated with dependence (assessed with the SDS) included taking higher than recommended doses, experiencing psychological distress, previous drug treatment, and chronic pain. ${ }^{33}$ A 1999 Canadian postal survey on prescribed and over-the-counter codeine (participants recruited via newspaper advertisements) found that $37 \%$ of respondents met DSM-IV criteria for codeine dependence, most of whom reported chronic pain and family histories of substance use problems. $^{20}$

\section{Case-control study}

A prospective case-control study described patients attending an addiction medicine clinic in China who were dependent on a codeine-containing cough syrup. ${ }^{37}$ This imaging study found that the patients, who exhibited increased impulsivity, had cortical white matter microstructural abnormalities. ${ }^{37}$

\section{Qualitative studies}

Three qualitative studies have examined the perceptions of pharmacists and codeine-dependent people. ${ }^{34,38,39}$ A British author $^{38}$ described the perception that dependence is not identified early, the challenges posed by the stigma attached to dependence, the fact that codeine-dependent people saw themselves as different to users of illicit opioids, and medical reasons for initiating codeine use. $^{38}$ A recent Irish study similarly described social stigma as a treatment barrier, and reported emotional distress as a driver for codeine use. $^{34}$

Two typologies of codeine dependence have been proposed: ${ }^{38,39}$ users who do not exceed therapeutic doses, and 
3 Individual patient reports: acute description or management of codeine-related harms

\begin{tabular}{|c|c|c|c|c|}
\hline Study & $\begin{array}{l}\text { Location of patient } \\
\text { (age, sex) }\end{array}$ & Harms from codeine use & Details of codeine dependence & Treatment \\
\hline $\begin{array}{l}\text { Faierman } \\
1973^{50}\end{array}$ & $\begin{array}{l}\text { USA } \\
\text { (male, 30) }\end{array}$ & $\begin{array}{l}\text { Hepatic injury with extensive fibrosis } \\
\text { attributed to terpin hydrate } \\
\text { component of cough syrup, as } \\
\text { opposed to codeine }\end{array}$ & $\begin{array}{l}16-20 \text { ounces codeine cough } \\
\text { syrup daily }\end{array}$ & Not reported \\
\hline $\begin{array}{l}\text { Wylie } \\
1994^{64}\end{array}$ & $\begin{array}{l}\text { Scotland } \\
\text { (female, 37) }\end{array}$ & Elevated liver enzyme levels & $\begin{array}{l}\text { Up to } 30 \times 500 \mathrm{mg} \text { paracetamol/ } \\
30 \mathrm{mg} \text { codeine phosphate per day }\end{array}$ & $\begin{array}{l}\text { Treated for potential hepatotoxicity } \\
\text { with acetylcysteine; discharged } \\
\text { after } 2 \text { days }\end{array}$ \\
\hline Dyer $2004^{47}$ & $\begin{array}{l}\text { United Kingdom } \\
\text { (male, 49) }\end{array}$ & $\begin{array}{l}\text { Perforated duodenal ulcer } 3 \text { years } \\
\text { previously (inappropriate ibuprofen } \\
\text { use), hypokalaemia }\end{array}$ & $\begin{array}{l}30 \times 200 \mathrm{mg} \\
\text { ibuprofen/12.8 mg } \\
\text { codeine in the } 3 \text { days before } \\
\text { admission. GP reported their taking } \\
24 \text { tablets at once previously }\end{array}$ & $\begin{array}{l}\text { Serum potassium corrected with } \\
\text { intravenous potassium therapy; } \\
\text { advised that Nurofen Plus misuse } \\
\text { caused his recurrent hypokalaemic } \\
\text { episodes; offered assistance }\end{array}$ \\
\hline \multirow[t]{2}{*}{$\begin{array}{l}\text { Dutch } \\
2008^{46}\end{array}$} & $\begin{array}{l}\text { Australia } \\
\text { (female, 39) }\end{array}$ & $\begin{array}{l}\text { Anterior gastric antrum ulcer and } \\
2.6 \mathrm{~L} \text { of green turbid fluid in the } \\
\text { peritoneal cavity }\end{array}$ & $\begin{array}{l}16-24 \times 200 \mathrm{mg} \text { ibuprofen/12.8 mg } \\
\text { codeine per day for } 3 \text { weeks }\end{array}$ & $\begin{array}{l}\text { Transferred to intensive care in } \\
\text { another hospital }\end{array}$ \\
\hline & $\begin{array}{l}\text { Australia } \\
\text { (male, 41) }\end{array}$ & $\begin{array}{l}\text { Gastric antrum ulcer with gross } \\
\text { abdominal contamination }\end{array}$ & $\begin{array}{l}\text { "A packet" of } 200 \mathrm{mg} \text { ibuprofen/ } \\
12.8 \mathrm{mg} \text { codeine per day for one year }\end{array}$ & $\begin{array}{l}\text { Postoperatively, patient offered } \\
\text { inpatient drug treatment, absconded } \\
\text { before transfer arranged }\end{array}$ \\
\hline \multirow[t]{3}{*}{$\begin{array}{l}\text { Karamatic } \\
2011^{53}\end{array}$} & $\begin{array}{l}\text { Australia } \\
\text { (male, 42) }\end{array}$ & $\begin{array}{l}\text { Multiple jejunal ulcers with early } \\
\text { structuring consistent with NSAID } \\
\text { enteropathy }\end{array}$ & $\begin{array}{l}10 \times 200 \mathrm{mg} \text { ibuprofen/12.8 mg } \\
\text { codeine phosphate per day }\end{array}$ & $\begin{array}{l}40 \mathrm{mg} \text { omeprazole, } 15 \mathrm{mg} \\
\text { mirtazapine daily; } 5 \mathrm{mg} \text { oxycodone } \\
\text { every } 4-6 \mathrm{~h} \text { as needed }\end{array}$ \\
\hline & $\begin{array}{l}\text { Australia } \\
\text { (female, 41) }\end{array}$ & $\begin{array}{l}\text { Multiple web-like strictures with } \\
\text { circumferential ulceration throughout } \\
\text { small bowel consistent with NSAID } \\
\text { enteropathy }\end{array}$ & $\begin{array}{l}20 \times 200 \mathrm{mg} \text { ibuprofen/12.8 mg } \\
\text { codeine phosphate per day, } 5 \text { years }\end{array}$ & Iron supplement \\
\hline & $\begin{array}{l}\text { Australia } \\
\text { (male, 41) }\end{array}$ & Multiple jejunal ulcers & $\begin{array}{l}10-12 \times 200 \mathrm{mg} \text { ibuprofen } / 12.8 \mathrm{mg} \\
\text { codeine phosphate per day, } \\
>5 \text { years }\end{array}$ & $\begin{array}{l}\text { Omeprazole and amitriptyline daily; } \\
\text { hyoscine butylbromide, paracetamol } \\
\text { and codeine and tramadol as } \\
\text { needed }\end{array}$ \\
\hline
\end{tabular}

$\mathrm{Ng} 2011^{58} \quad$ Australia

(female, 32)

Australia

(male, 37)

Australia

(female, 45)

Australia

(male, 40)

Page $2011^{59}$ Australia

(females, 35, 39;

males, 41, 55)

Lake $2013^{56}$ Australia

(male, 35)

$\begin{array}{lll}\begin{array}{l}\text { Roussin } \\ 2013^{61}\end{array} & \begin{array}{l}\text { France } \\ \text { (females, 38, 38, 42, } \\ \text { 47; males, 42, 55) }\end{array} & \begin{array}{l}\text { Included depressive mood, and } \\ \text { constipation and vertigo }\end{array} \\ \begin{array}{l}\text { Ammit } \\ 2016^{44}\end{array} & \begin{array}{l}\text { Australia } \\ \text { (female, 39) }\end{array} & \begin{array}{l}\text { Gastric erosion and renal tubular } \\ \text { acidosis }\end{array}\end{array}$
without nephrocalcinosis

Progressive muscle weakness, low tubular acidosis stricture tubular acidosis gap metabolic acidosis NSAID, loss of partner and employment
Oesophageal erosions, benign gastric $20 \times 200 \mathrm{mg}$ ibuprofen/12.8 mg ulcer, enlarged, oedematous kidneys codeine phosphate per day serum potassium level, biochemical features consistent with distal renal

Microcytic anaemia, gastric antral $\quad 50 \times 200 \mathrm{mg}$ ibuprofen/12.8 mg ulceration with a peptic oesophageal codeine phosphate per day

Generalised weakness associated $\quad 1.4-2.0 \mathrm{~g}$ ibuprofen in codeine with hypokalaemia, with distal renal combination product per day for

3 months

Renal tubular acidosis, normal anion Longstanding misuse of ibuprofen

Small bowel stricture secondary to

(5-18 g/day) and codeine (320-1152 mg/day) in over-thecounter medications

Up to $90 \times 200 \mathrm{mg}$ ibuprofen/ $12.8 \mathrm{mg}$ codeine phosphate per day, bowel resection; patient controlled clear salience and neuroadaptation concomitant ketamine use;

120-200 mg codeine phosphate in combination product with paracetamol per day for $1-10$ years

$520 \mathrm{mg} /$ day (over-the-counter ibuprofen combination) for past year; increased with physical and psychological stress community drug treatment on discharge

\section{Electrolyte replacement}

Electrolyte replacement, buprenorphine maintenance therapy

Electrolyte replacement

Electrolyte replacement

Biochemical recovery of all patients; two patients required intensive care admission for central venous access and potassium replacement

\section{Not reported}

\section{Symptomatic medication}

(diazepam, paracetamol, baclofen): balloon enteroscopy/dilation (small bowel obstruction); education about harms of ibuprofen. Following admission: opioid substitution therapy, counselling and 12-step program 
4 Individual patient reports: treatment of codeine dependence, with or without management of acute harms

\begin{tabular}{llll} 
Study & $\begin{array}{l}\text { Location } \\
\text { (sex, age) }\end{array}$ & Harms from codeine use & Details of codeine dependence \\
\hline Gruber 1948 & USA & $\begin{array}{l}\text { Neuroadaptation and decline in } \\
\text { functioning, loss of weight, likely }\end{array}$ & $\begin{array}{l}\text { Intravenous codeine up to } 4.8 \mathrm{~g} \\
\text { daily in weeks before }\end{array}$ \\
& $\begin{array}{l}\text { maintenance of chronic pain } \\
\text { symptoms, suicide following }\end{array}$ & $\begin{array}{l}\text { hospitalisation; } 660 \mathrm{mg} \text { per day } \\
\text { in previous months }\end{array}$ \\
& $\begin{array}{ll}\text { withdrawal } \\
\end{array}$ &
\end{tabular}

Treatment and outcome

Reducing doses of intravenous codeine over 12 days; $100 \mathrm{mg}$ pethidine iv 4-6 times per day (days 3 $-11)$, acetylsalicylic acid injections (days 12-15)

Withdrawal syndrome tolerated with some discomfort; committed suicide after discharge

$\begin{array}{rlr}\text { Vaughan } 1967^{63} & \begin{array}{l}\text { New Zealand } \\ \text { (male, 53) }\end{array} & \text { Renal failure (fatal) } \\ \begin{array}{l}\text { New Zealand } \\ \text { (female, 39) }\end{array} & \text { Renal failure (fatal) } \\ \begin{array}{l}\text { New Zealand } \\ \text { (female, 70) }\end{array} & \text { Analgesic nephropathy } \\ \begin{array}{l}\text { New Zealand } \\ \text { (male, 28) }\end{array} & \begin{array}{l}\text { Possible medication overuse } \\ \text { headache }\end{array}\end{array}$

8-12 aspirin/phenacetin/codeine tablets daily, several years

50 aspirin/phenacetin/codeine tablets per week

8 aspirin/phenacetin/codeine tablets per day, 20 years

6-20 aspirin/phenacetin/codeine tablets per day, 20 years

10-year history of codeine use

$\begin{array}{lll}\text { Senjo 198962 } & \text { Japan } & \begin{array}{l}\text { Suspected codeine use } \\ \text { (male, 34) }\end{array} \\ & \begin{array}{l}\text { contributed to } \\ \text { obsessive-compulsive } \\ \text { disorder in both patients }\end{array}\end{array}$

Japan

(male, 35)

10-year history of codeine use

\section{Supportive therapy}

Symptomatic treatment for renal failure

Education on link between symptoms and analgesic use; patient ceased analgesics

Inpatient stay (2 months); withdrawal symptoms after 5 days codeine-free Obsessive-compulsive disorder improved after withdrawal

Patient was violent after 2 days, transferred to another hospital Returned 2 months later with complete remission of symptoms

Bedi $1991^{45} \quad$ India Dependence and opioid
(male, 42) withdrawal symptoms

Two bottles Phensedyl (total Loperamide, diazepam, nitrazepam; content: $450 \mathrm{mg}$ codeine, $366 \mathrm{mg}$ supportive psychotherapy and family ephedrine, $180 \mathrm{mg}$ promethazine) per day

counselling advocated; drugs reduced over 10 days

$\begin{array}{llll}\text { Eng } 1996^{48} & \text { USA } & \text { Medication overuse headache } & \begin{array}{l}6-15 \times \text { paracetamol/codeine } \\ \text { (male, 54) }\end{array}\end{array}$

Lake $2008^{55} \quad$ USA Transformation of episodic to (female, 39) daily headache

10 butalbital with codeine and acetaminophen tablets per day for pain control for past year

$\begin{array}{lll}\text { Evans 201049 } & \begin{array}{l}\text { New Zealand } \\ \text { (male, 35) }\end{array} & \begin{array}{l}\text { Acute gastric ulcer, severe } \\ \text { gastritis and post-bulbar } \\ \text { duodenitis with active bleedin }\end{array} \\ \text { Robinson } 2010^{60} & \begin{array}{l}\text { New Zealand } \\ \text { (male, 53) }\end{array} & \begin{array}{l}\text { Gastric ulcer, gastric bleeding, } \\ \text { hepatotoxicity }\end{array}\end{array}$

New Zealand (female, 31)

New Zealand (female, 63)

New Zealand (female, 47)
Peptic ulcer and anaemia

Gastric ulcer

"Inflammatory bowel disease"
More than $100 \times 200 \mathrm{mg}$ ibuprofen/12.8 mg codeine phosphate per day for back pain

$60-80 \times 200$

mg ibuprofen/12.8 mg codeine phosphate per day, 2 years

$48 \times 200 \mathrm{mg}$ ibuprofen/12.8 mg codeine phosphate per day, 2 years

$20 \times 200 \mathrm{mg}$ ibuprofen/12.8 mg codeine phosphate (and prescription codeine) per day, 3 years

Up to $72 \times 200 \mathrm{mg}$ ibuprofen/ $12.8 \mathrm{mg}$ codeine phosphate per day, one year
Detoxification (methadone); referral to anxiety disorders program (diagnosed with GAD), CBT, taught relaxation

Self-managed analgesic use (reduced analgesic use to paracetamol twice a week or less), developed alternative strategies for psychological symptoms

Withdrawn from butalbital, codeine; coached in relaxation techniques. After multiple admissions: weekly psychotherapy, formal substance abuse program, observed urine drug screens). CBT, pain

management.12-step program Ongoing relapse, eventually ceased substance use, diagnosed with fibromyalgia and prescribed opioids

Reducing codeine dose prescribed; counselling

Gastrointestinal symptoms healed, but balloon dilation of pyloric stenosis required

Treatment not reported

Many patients reported significant opioid withdrawal symptoms despite treatment with ancillary medications 
4 Individual patient reports: treatment of codeine dependence, with or without management of acute harms (continued)

\begin{tabular}{|c|c|c|c|c|}
\hline Study & $\begin{array}{l}\text { Location } \\
\text { (sex, age) }\end{array}$ & Harms from codeine use & Details of codeine dependence & Treatment and outcome \\
\hline & $\begin{array}{l}\text { New Zealand } \\
\text { (female, } 31 \text { ) }\end{array}$ & $\begin{array}{l}\text { Gastric ulcer and bleeding } \\
\text { (previous gastrectomy) }\end{array}$ & $\begin{array}{l}\text { Up to } 120 \times 200 \mathrm{mg} \\
\text { ibuprofen/12.8 mg codeine } \\
\text { phosphate per day, } 2 \text { years }\end{array}$ & \\
\hline Hard $2014^{52}$ & $\begin{array}{l}\text { United Kingdom } \\
\text { (female, mid-20s) }\end{array}$ & $\begin{array}{l}\text { Neuroadaptation, exclusion of } \\
\text { other activities, financial }\end{array}$ & $\begin{array}{l}\text { 10-year history of codeine } \\
\text { dependence (initially prescribed) }\end{array}$ & $\begin{array}{l}\text { GP changed from codeine to } \\
\text { dihydrocodeine as a harm } \\
\text { minimisation strategy } \\
\text { (approximately } 2940 \mathrm{mg} \\
\text { dihydrocodeine daily). Buprenorphine/ } \\
\text { naloxone (maintenance: } \\
10 \mathrm{mg} \text { buprenorphine/2.5 mg } \\
\text { naloxone); engaged with recovery } \\
\text { support services and psychosocial } \\
\text { counselling; } 12 \text {-step program } \\
\text { Initially mild precipitated withdrawal; } \\
\text { stabilised and returned to work }\end{array}$ \\
\hline Marr $2015^{57}$ & $\begin{array}{l}\text { Scotland } \\
\text { (female, 24) }\end{array}$ & Dependence & $\begin{array}{l}\text { Initially self-medication for dental } \\
\text { pain, escalated to prescribed and } \\
\text { over-the-counter opioids }\end{array}$ & $\begin{array}{l}\text { Stabilised on } 16 \text { mg buprenorphine/ } \\
4 \text { mg naloxone, transferred to mono- } \\
\text { product for pregnancy } \\
\text { Transferred to community } \\
\text { prescriber, reported stigma and } \\
\text { discomfort with drug treatment } \\
\text { clinic environment }\end{array}$ \\
\hline
\end{tabular}

Kean $2016^{54} \quad$ United Kingdom Neuroadaptation, relationship (male, mid-30s) disharmony, rebound headaches, hypoalbuminaemia, ALT levels elevated
Codeine prescribed by GP for back Buprenorphine/naloxone induction pain, later supplemented with illicit (up to $8 \mathrm{mg} / 2 \mathrm{mg}$ daily), tapered over codeine, escalating over years to 4 months. Anxiety/depression at end $250 \mathrm{mg} /$ day

of taper responded to fluoxetine, counselling

Abstinent, functioning and intact relationships

Van Hout $2016^{65}$ Ireland (female, 57)

Estranged from family, unable to work, episode of haematemesis

Ireland (female, 44)

Identified because of high volume of sick notes (impact on employment)

Ireland

(male, 45)

Perforated ulcer requiring surgical repair, three later ulcers, multiple surgical admissions for epigastric pain and gastrointestinal bleeding

Long history of over-the-counter codeine misuse causing lifethreatening morbidity
Use escalated from 12 to $24-48$ tablets per day over 3 years after fracture
Stabilised on $4 \mathrm{mg}$ buprenorphine/1 $\mathrm{mg}$ naloxone, counselling every 2 weeks Continues treatment in pharmacy setting; plan after 2 years to begin taper

Escalated use of over-the-counter Commenced buprenorphine-naloxone codeine (about 36 tablets per day) venlafaxine for depression, propranolol at time of traumatic event for migraine and omeprazole for a peptic ulcer

Stabilised on a $14 \mathrm{mg}$ buprenorphine/ $3.5 \mathrm{mg}$ naloxone, migraines largely resolved; soon after treatment, antidepressant treatment ended. Returned to work, planned reduction of buprenorphine

Several failed detoxifications; attempts to stabilise on maintenance dose of codeine failed. Prescribed buprenorphine/naloxone Overdosed on benzodiazepines, hospitalised, buprenorphine withdrawn. After restabilisation, maintained on $12 \mathrm{mg}$ buprenorphine/3 $\mathrm{mg}$ naloxone

Ireland

Not specifically reported

(male, 44)

Escalating amounts of over-the-

Buprenorphine-naloxone,

counter codeine-ibuprofen (up to psychosocial interventions. Initial 72 tablets per day) over several relapse (attempt to self-detoxify), years

recommenced on higher dose

Initially stabilised on maintenance dose of $8 \mathrm{mg}$ buprenorphine $/ 2 \mathrm{mg}$ naloxone daily; recommenced and stabilised on $12 \mathrm{mg}$ buprenorphine/3 mg naloxone daily, ongoing counselling 
codeine-dependent people who consume high doses of the drug. The second group is characterised by severe dependence and harms. Additional typologies include recreational users ${ }^{39}$ and people who slightly exceed recommended doses. ${ }^{38}$

\section{Retrospective chart reviews and case series}

Five case series or retrospective chart reviews ${ }^{19,40-43}$ identified common features of people with codeine dependence, including higher proportions of women than among those treated for misuse of other opioids, histories of problematic alcohol use, mental health comorbidity, and serious side effects resulting from using combination medicines containing codeine, including one death. ${ }^{41}$ Prior heroin dependence was rare.

\section{Reports on individual patients}

Twenty-two reports described 49 individuals who were codeinedependent $^{44-65}$ (Box 3, Box 4; online Appendix, tables 4B and 4C). Twenty-three were women (mean age, 42 years [SD, 9 years]). Of the 15 people for whom data on employment status were reported, nine were employed. Acute harms and information on treatment approaches were described. Inherent to cases of acute harm were complications attributable to the co-medications paracetamol and ibuprofen, including distal renal tubular acidosis, hypokalaemia, gastritis and other enteropathies, medication overuse headache, hepatic necrosis, hypoalbuminaemia, microcytic anaemia, and weight loss. In a case series of 27 patients, ${ }^{19}$ most had initiated codeine to treat pain but later escalated their intake for other reasons; this was also reported in many case reports.

Acute management of harms was characterised by inpatient hospital management of serious problems (often requiring intensive care) to restore electrolyte balance, ${ }^{47,58,59}$ manage gastrointestinal symptoms ${ }^{44,53}$ (including bowel resection), ${ }^{56}$ and to assess or treat hepatotoxicity. ${ }^{50,64}$ Opioid withdrawal was managed with symptomatic medications and buprenorphine; potential hepatotoxicity was managed with acetylcysteine. ${ }^{64}$

Psychiatric comorbidity in people with codeine dependence was dominated by high prevalence conditions (depression and anxiety disorders). ${ }^{20,27,44,48,53,58,60,61}$ Some reports described prior or comorbid addictions (benzodiazepines, opioids, alcohol), $34,35,40,42,60$ and mental health conditions. ${ }^{20,40-42,60}$ Bipolar disorder, ${ }^{27,42}$ obsessive-compulsive disorder, ${ }^{62}$ relationship breakdown, ${ }^{47}$ suicide behaviour, ${ }^{51,56}$ and bereavement or loss ${ }^{47}$ were also described.

Individual patient reports described treatment approaches, including attempted self-management, ${ }^{45,65}$ use of symptomatic medications, ${ }^{60}$ prescribed codeine or dihydrocodeine ${ }^{49,52}$ or buprenorphine and naloxone,,$^{52,54,65}$ and detoxification with methadone. ${ }^{48}$ Management of mental health symptoms with antidepressants and behavioural therapies was described. ${ }^{48,54,55,65}$ One notable case combined methadone taper, cognitive behavioural therapy, and relaxation strategies, enabling codeine cessation and self-management of pain. ${ }^{48}$ Resolution of presenting complaints (obsessive-compulsive disorder, medication overuse headache) after codeine cessation was described. ${ }^{62,63}$

\section{Treatment studies}

Ten studies described treatment approaches in detail (Box 5; online Appendix, table 4D). Common medication-based approaches included taper from codeine with symptomatic medications such as clonidine or benzodiazepines, ${ }^{42,67}$ buprenorphine maintenance, ${ }^{19,27}$ CYP inhibitors, ${ }^{66,68}$ and gradual self-managed taper. ${ }^{38}$ Positive outcomes for opioid agonist treatment (methadone and buprenorphine with or without naloxone) were described. ${ }^{19,27,34,38,40,43,69}$ The role of internet support groups ${ }^{38}$ and psychosocial treatments, including cognitive behavioural therapy, were highlighted in some studies. ${ }^{42,67,69}$

Two studies tested the hypothesis that preventing the O-demethylation of codeine to morphine with CYP inhibitors would reduce codeine use. ${ }^{66,68}$ Initial promising results from an open label pilot study of fluoxetine (14 subjects) ${ }^{66}$ were not replicated in a small randomised controlled trial that compared the effectiveness of fluoxetine or quinidine (two potent CYP2D6 inhibitors) with placebo. ${ }^{68}$

A retrospective review of inpatient admissions described taper with clonidine and benzodiazepines, combined with an intensive 4-week mental health treatment program. ${ }^{42}$ Patients had a mean stay of 42 days (SD, 23 days), with withdrawal symptoms requiring treatment for a mean 16 days (SD, 10 days). Taper with buprenorphine was described by an Australian study which noted that codeine dependence was more likely to be treated with taper rather than maintenance. ${ }^{27}$ Relapse after taper was not uncommon. ${ }^{34,42}$

A single arm study (11 patients) found that cognitive behavioural therapy could significantly reduce codeine use - six patients ceased using codeine altogether - and neuropsychological functioning was improved by codeine reduction or cessation without deterioration in pain or quality of life. ${ }^{67}$ A mixed methods study also highlighted the role of psychological therapies. ${ }^{69}$

Seven studies described treatment with opioid agonists, ${ }^{19,27,34,38,40,43,69}$ four of which did not report outcomes. ${ }^{19,34,40,69}$ One small retrospective cohort study described high retention rates for patients treated with buprenorphine over 28 days (median daily dose, $12-16 \mathrm{mg}$ ); one patient described initial sedation that necessitated reducing the dose. ${ }^{43}$

Two qualitative studies described positive experiences and outcomes for treatment with methadone and buprenorphine, despite patient concerns about the treatment experience and the clinic environment. ${ }^{34,38}$

According to GRADE criteria, the quality of evidence from treatment studies was very low to low; most studies were retrospective and descriptive, and all had small sample sizes.

\section{Discussion}

Our review of codeine-dependent people indicates that approximately equal proportions of men and women are involved; their mean age is greater than for patients treated for problematic use of other opioids, the prevalence of mental health comorbidity is high, identification of dependence is often delayed, and patients experience serious complications associated with excessive consumption of combination products that include codeine. Problematic codeine use was associated with mental health problems. The quality and methodology of the studies we assessed varied considerably, but their depictions of the features associated with codeine dependence were consistent, describing a clinically challenging area in which under-reporting is highly likely. The reports highlight the importance of asking about the use of nonprescribed analgesics in a range of health care situations, particularly when gastrointestinal complications are identified. The diversity of those affected and the high level of morbidity suggest that population level interventions are required for screening and prevention wherever codeine is available over the counter. Careful questioning about recent patterns of use, the reasons for taking codeine, and withdrawal symptoms upon cessation may help identify when a patient should be comprehensively assessed for an opioid use disorder. 


\section{Treatment approaches for codeine dependence}

$\begin{array}{llll}\text { Study } & \text { Design, location } & \text { Sample size (sex), age } & \text { Treatment approach and outcomes }\end{array}$

$\begin{array}{lll}\text { Fernandes } & \text { Double blind, } & 30 \text { assessed, 17 started } \\ 2002^{68} & \text { randomised } & \text { treatment (65\% female) } \\ & \text { controlled trial, } & \text { mean, 40 years (SD, } 12 \\ \text { Canada } & \text { years) }\end{array}$

All patients received brief behavioural therapy. Two Low weeks of baseline monitoring were followed by 8 weeks of daily treatment with fluoxetine or quinidine (two potent CYP2D6 inhibitors) or placebo No significant difference among the three groups in daily codeine intake or depression scores. By end of treatment, large decrease from baseline in mean daily codeine use in all groups: placebo by $57 \%$, quinidine by $56 \%$, and fluoxetine by $51 \%$

\begin{tabular}{|c|c|c|c|c|}
\hline Frei $2010^{19}$ & Case series, Australia & $\begin{array}{l}27 \text { ( } 48 \% \text { female); } 20 \text { years } \\
\text { or more }\end{array}$ & $\begin{array}{l}\text { Opioid pharmacotherapy ( } 16 \text { patients), } \\
\text { buprenorphine taper ( } 3 \text { ), buprenorphine } \\
\text { maintenance (10), methadone (3). Outcomes not } \\
\text { reported }\end{array}$ & Very low \\
\hline Thekiso $2010^{42}$ & $\begin{array}{l}\text { Retrospective chart } \\
\text { review, Ireland }\end{array}$ & $\begin{array}{l}20 \text { ( } 65 \% \text { female): mean, } \\
49.2 \text { years (SD, } 23.4 \text { years) }\end{array}$ & $\begin{array}{l}\text { Treated for substance withdrawal with standard } \\
\text { pharmacological protocol-driven regimes, } \\
\text { underwent up to } 4 \text { weeks' comprehensive inpatient } \\
\text { treatment. Withdrawal regime included tapering } \\
\text { benzodiazepines and clonidine. Affective } \\
\text { comorbidities also treated (pharmacological, } \\
\text { "psycho-education" } \\
\text { Mean length of stay, } 42 \text { days; mean length of } \\
\text { treatment for withdrawal, } 16 \text { days }\end{array}$ & Very low \\
\hline Nielsen $2015^{27}$ & $\begin{array}{l}\text { Retrospective chart } \\
\text { review, Australia }\end{array}$ & $\begin{array}{l}135 \text { ( } 53 \text { codeine-dependent, } \\
66 \% \text { women; } \\
\text { mean, } 38.6 \text { years) }\end{array}$ & $\begin{array}{l}\text { Codeine dependence more likely to be treated with } \\
\text { buprenorphine than methadone, withdrawal } \\
\text { management more common than longer term } \\
\text { pharmacotherapy. Outcomes not reported }\end{array}$ & Very low \\
\hline Nielsen $2015^{43}$ & $\begin{array}{l}\text { Retrospective chart } \\
\text { review, Australia }\end{array}$ & $\begin{array}{l}19 \text { ( } 84 \% \text { female); mean, } 41 \\
\text { years (SD, } 9 \text { years) }\end{array}$ & $\begin{array}{l}\text { Buprenorphine maintenance treatment by drug } \\
\text { treatment services, five as inpatients,14 as } \\
\text { outpatients } \\
\text { Median dose, } 12-16 \mathrm{mg} \text { buprenorphine, four patients } \\
\text { continued to use opioids. buprenorphine doses } \\
\text { higher than estimated based on codeine dose }\end{array}$ & Very low \\
\hline Norman $2016^{69}$ & $\begin{array}{l}\text { Mixed methods } \\
\text { (systematic review, } \\
\text { qualitative } \\
\text { interviews), } \\
\text { Ireland, United } \\
\text { Kingdom, } \\
\text { South Africa }\end{array}$ & $\begin{array}{l}23 \text { interviews with key } \\
\text { experts }\end{array}$ & $\begin{array}{l}\text { Buprenorphine and methadone in substitution } \\
\text { therapy. Notes efficacy of CBT for treating } \\
\text { opioid dependence } \\
\text { Outlined "best practices" in treatment reported by } \\
\text { stakeholders, suggested "innovations" for treatment. } \\
\text { Did not assess treatments }\end{array}$ & Very low \\
\hline
\end{tabular}


Treatment approaches include self-management with internet support, psychological treatments, symptomatic medications for opioid withdrawal, and opioid agonist treatments. In particular, buprenorphine treatment undertaken according to current guidelines was commonly described. Studies of opioid taper found that relapse was common (consistent with taper for opioid dependence in general). Taken together, the treatment studies and case reports provide evidence that opioid agonist treatments, combined with psychosocial adjuncts, may be suitable and acceptable to patients. The evidence, albeit low in quality, indicates that positive treatment outcomes could be achieved with these approaches.

In the absence of specific high quality evidence, judgements about approaches for treating people with codeine dependence must be based largely on studies of opioid dependence. The effectiveness of treatment with methadone and buprenorphine has been reported, and maintenance is more effective than withdrawal and detoxification for people who are dependent on pharmaceutical opioids ${ }^{70}$ or opioids in general. ${ }^{71}$ Research on selecting patients for treatment with opioid agonists is limited. According to the general principles of treatment, diagnosis of opioid dependence must first be confirmed. ${ }^{72}$ A stepped care approach with less intensive treatment (eg, taper, counselling) for low severity dependence is recommended by national guidelines. ${ }^{72}$ Patients who unsuccessfully attempt taper may be considered for maintenance opioid agonist treatment, which achieves better treatment outcomes than detoxification for pharmaceutical opioid dependence. ${ }^{71}$ Because of wide variations in codeine metabolism, predicting opioid requirements with dose conversion tables is challenging; ${ }^{43}$ for this reason their use is discouraged.

Psychological adjunct therapies can be beneficial, ${ }^{73}$ but the role of psychosocial interventions as accompaniments to opioid agonist treatments requires further research. ${ }^{74}$ The high prevalence of mental health comorbidities and the preference of patients for online support may indicate that online interventions for managing comorbidity may be useful. In general, the role of pharmacological treatments for depression or anxiety at the start of treatment is unclear. It is recommended that comorbidities are assessed after a period of abstinence because of the potential for diagnostic uncertainty caused by the acute effects of opioid toxicity and withdrawal. ${ }^{75}$

The treatment setting is also important. People consuming larger amounts of opioids together with sedatives (eg, benzodiazepines) are a population at greater risk, and referral to a specialist may be required. ${ }^{72}$ Characteristics that may indicate that patients are appropriate for managing in primary care include being employed, having social support, and not having another substance use disorder or a history of illicit drug use.

\section{Medication overuse headache}

Headache is a common reason for initiating codeine use by patients who develop dependence. ${ }^{19,61}$ Paradoxically, medication overuse headache - in this context, exacerbation of a pre-existing headache disorder by excessive intake of codeine is another potential complication of codeine dependence. ${ }^{48,55,63}$ Data that might guide the management of codeine overuse headache specifically have not been published. Management of opioid-related medication overuse headache usually consists of patient education, opioid withdrawal, and the initiation of prophylactic agents, ${ }^{76-78}$ often in an inpatient setting. ${ }^{76}$ Medication overuse headache that results from overusing analgesics, compared with overuse of triptans, is associated with a greater withdrawal headache duration (about 10 days), ${ }^{79}$ with meaningful improvement only after 12 weeks or more, ${ }^{80}$ and high relapse rates (eg, $71 \%$ at 4 years ${ }^{81}$ ).

\section{Limitations of our analysis}

Comparing codeine dependence in different groups of patients was made difficult by changing usage patterns over time, subgroup heterogeneity, and probable under-reporting of codeine use. Methodological constraints included low participant numbers, selection biases (admissions, help-seeking or co-medication sequelae as a proxy for neuroadaptation to codeine), and a lack of objective and standardised criteria for determining codeine dependence. Many studies employed internet-based recruitment or data collection, ${ }^{33,38}$ potentially limiting the generalisability of findings to users without regular internet access, but this might be offset by the ability to reach users who are otherwise difficult to reach. Some studies did not specify whether codeine was prescribed or obtained over the counter, but most reports were concerned with over-the-counter codeine. Many studies that included codeine-dependent people were excluded from our analysis because they did not separately describe codeine dependence; this particularly applied to studies of medication overuse headache. Nevertheless, our review is the most comprehensive synthesis of data on the phenomena of codeine dependence, and we have described a range of potential treatment responses, including medication- and non-medication-based treatments.

\section{Conclusion}

Codeine dependence can be identified by screening patients who present with acute complications associated with taking combination analgesics, and by routine questioning about over-thecounter medication use. Common treatment approaches include detoxification and opioid agonist treatment. Clinical leadership in providing guidance about how to identify and treat individuals with codeine dependence is required as a matter of public health.

Acknowledgements: Suzanne Nielsen holds a National Health and Medical Research Council Research Fellowship (1132433).

Competing interests: Suzanne Nielsen is a named investigator on untied educational grants from Reckitt-Benckiser and Indivior. Tim MacDonald has received honoraria, fees and professional development resources from Servier, the Australian and New Zealand Mental Health Association, and Healthe Care; he works in the private sector and receives income for clinical services.

Provenance: Not commissioned; externally peer reviewed.

C 2018 AMPCo Pty Ltd. Produced with Elsevier B.V. All rights reserved.
1 International Narcotics Control Board. Narcotic drugs estimated world requirements for 2015 - statistics for 2013. New York: International Narcotics Control Board, 2015. https://www.incb.org/documents/Narcotic-Drugs/ Technical-Publications/2014/Narcotic Drugs_Report 2014.pdf (viewed Apr 2017).

2 Gisev N, Nielsen S, Cama E, et al. An ecological study of the extent and factors associated with the use of prescription and over-the-counter codeine in Australia. Eur J Clin Pharmacol 2016; 72: 469-494.

3 Van Hout MC, Bergin M, Foley M, et al. A scoping review of codeine use, misuse and dependence; final report. Brussels: CODEMISUSED Project European Commission 7th Framework Programme, 2014. http://codemisused. org/uploads/files/Van_Hout_et_al_Scoping_Report_2303-2015.pdf (viewed Apr 2017)
4 Coller JK, Christrup LL, Somogyi AA. Role of active metabolites in the use of opioids. Eur J Clin Pharmacol 2009; 65: 121-439.

5 ledema J. Cautions with codeine. Aust Prescr 2011; 34: 133-135.

6 Somogyi AA, Barratt DT, Coller JK. Pharmacogenetics of opioids. Clin Pharmacol Ther 2007; 81: 429-444. 
7 Mattoo SK, Basu D, Sharma A, et al. Abuse of codeinecontaining cough syrups: a report from India. Addiction 1997; 92: 1783-1787.

8 Orriols L, Gaillard J, Lapeyre-Mestre M, Roussin A. Evaluation of abuse and dependence on drugs used for self-medication: a pharmacoepidemiological pilot study based on community pharmacies in France. Drug Saf 2009; 32: 859-873.

9 Nielsen S, Cameron J, Pahoki S. Over the counter codeine dependence [report]. Melbourne: Turning Point Alcohol and Drug Centre, 2010. http://atdc.org.au/wp-content/ uploads/2011/02/OTC_CODEINE_REPORT.pdf (viewed Dec 2017).

10 Murnion BP. Combination analgesics in adults. Aust Prescr 2010; 33: 113-115.

II McQuay HJ, Carroll D, Watts PG, et al. Does adding small doses of codeine increase pain relief after third molar surgery? Clin J Pain 1986; 2: 197-202.

12 Zhang WY, Po A. Do codeine and caffeine enhance the analgesic effect of aspirin? A systematic overview. J Clin Pharm Ther 1997; 22: 79-97.

13 Nielsen S, Tobin CL, Dobbin MDH. OTC codeine: examining the evidence for and against. Aust Pharmacist 2012; (Mar): 236-240.

14 Baratta JL, Gandhi K, Viscusi ER. Limited evidence that single-dose oral ibuprofen plus codeine is more effective than either drug alone. Evid Based Nurs 2014; 17: 51-52.

15 Derry S, Karlin SM, Moore R. Single dose oral ibuprofen plus codeine for acute postoperative pain in adults. Cochrane Database Syst Rev 2015; (2): CD01017.

16 MacDonald N, MacLeod SM. Has the time come to phase out codeine? CMAJ 2010; 182: 1825.

17 Anderson BJ. Is it farewell to codeine? Arch Dis Child 2013; 98: 986-988

18 Babalonis S, Lofwall MR, Nuzzo PA, et al. Abuse liability and reinforcing efficacy of oral tramadol in humans. Drug Alcohol Depend 2013; 129: 116-124.

19 Frei MY, Nielsen S, Dobbin MD, Tobin CL. Serious morbidity associated with misuse of over-the-counter codeine-ibuprofen analgesics: a series of 27 cases. Med J Aust 2010; 193: 294-296. https://www.mja.com.au/ journal/2010/193/5/serious-morbidity-associatedmisuse-over-counter-codeine-ibuprofen-analgesics

20 Sproule BA, Busto UE, Somer G, Romach MK. Characteristics of dependent and nondependent regular users of codeine. J Clin Psychopharmacol 1999; 19: 367-372.

21 Mill D, Johnson JL, Cock V, et al. Counting the cost of over-the-counter codeine containing analgesic misuse: a retrospective review of hospital admissions over a 5 year period. Drug Alcohol Rev 2017; doi: 10.1111/dar.12595 [Epub ahead of print].

22 Pilgrim JL, Dobbin M, Drummer OH. Fatal misuse of codeine-ibuprofen analgesics in Victoria, Australia. Med J Aust 2013; 199: 329-331. https://www.mja.com.au/ journal/2013/199/5/fatal-misuse-codeine-ibuprofenanalgesics-victoria-australia

23 Australian Government Department of Health, Therapeutic Goods Administration. Scheduling delegate's final decision: codeine, December 2016. Updated Jan 2017. http://www.tga.gov.au/schedulingdecision-final/scheduling-delegates-final-decisioncodeine-december-2016 (viewed Dec 2017).

24 Manitoba College of Pharmacists. Practice direction: exempted codeine preparations [effective Feb 2016]. http://mpha.inltouch.org/uploaded/web/Legislation/ Practice\%20Resources/Exempted\%20Codeine\% 20Products\%20Council\%20Approved.pdf (viewed May 2017).

25 Cracknell C. Codeine now restricted to prescription-only. The Connexion [website] 13 July 2017. https://www.connexionfrance.com/Frenchnews/Codeine-now-restricted-to-prescription-only (viewed July 2017).
26 Myers B, Siegfried N, Parry CD. Over-the-counter and prescription medicine misuse in Cape Town: findings from specialist treatment centres. S Afr Med J 2003; 93 : 367-370.

27 Nielsen S, Murnion B, Dunlop A, et al. Comparing treatment-seeking codeine users and strong opioid users: findings from a novel case series. Drug Alcohol Rev 2015; 34: 304-311.

28 Nielsen S, Roxburgh A, Bruno R, et al. Changes in non-opioid substitution treatment episodes for pharmaceutical opioids and heroin from 2002 to 2011. Drug Alcohol Depend 2015; 149: 212-219.

29 Joanna Briggs Institute. Critical appraisal checklist for analytical cross sectional studies. Adelaide: Joanna Briggs Institute, 2016. http://joannabriggs-webdev.org/ assets/docs/critical-appraisal-tools/JBI_Critical_ Appraisal-Checklist_for_Analytical_Cross_Sectional_ Studies.pdf (viewed Apr 2017).

30 Glynn L. A critical appraisal tool for library and information research. Library Hi Tech 2006; 24: 387-339.

31 Guyatt G, Oxman A, Akl E, et al. GRADE guidelines. Introduction: GRADE evidence profiles and summary of findings tables. J Clin Epidemiol 2011; 64: 383-394.

32 Gossop M, Darke S, Griffiths P, et al. The Severity of Dependence Scale (SDS): psychometric properties of the SDS in English and Australian samples of heroin, cocaine and amphetamine users. Addiction 1995; 90: 607-614.

33 Nielsen S, Cameron J, Lee N. Characteristics of a nontreatment-seeking sample of over-the-counter codeine users: implications for intervention and prevention. J Opioid Manag 2011; 7: 363-370.

34 Van Hout MC, Horan A, Santlal K, et al. "Codeine is my companion": misuse and dependence on codeine containing medicines in Ireland. Ir J Psychol Med 2015; 9: 1-14.

35 Fredheim O, Skurtveit S, Moroz A, et al. Prescription pattern of codeine for non-malignant pain: a pharmacoepidemiological study from the Norwegian Prescription Database. Acta Anaesthesiol Scand 2009; 53: 627-633.

36 Dada S, Burnhams NH, Van Hout MC, Parry CDH. Codeine misuse and dependence in South Africa: learning from substance abuse treatment admissions. S Afr Med J 2015; 105: 776-779.

37 Qiu YW, Su HH, Lv XF, Jiang GH. Abnormal white matter integrity in chronic users of codeine-containing cough syrups: a tract-based spatial statistics study. AJNR Am J Neuroradiol 2015; 36: 50-56.

38 Cooper RJ. "I can' t be an addict. I am." Over-the-counter medicine abuse: a qualitative study. BMJ Open 2013; 3: e002913.

39 Nielsen S, Cameron J, Pahoki S. Opportunities and challenges: over-the-counter codeine supply from the codeine consumer's perspective. Int J Pharm Pract 2013; 21: 161-168.

40 McAvoy BR, Dobbin MD, Tobin CL. Over-the-counter codeine analgesic misuse and harm: characteristics of cases in Australia and New Zealand. N Z Med I 2011; 124: 29-33.

41 McDonough MA. Misuse of codeine-containing combination analgesics. Med J Aust 2011; 194: 486. https://www.mja.com.au/journal/2011/194/9/misusecodeine-containing-combination-analgesics

42 Thekiso TB, Farren C. Over the counter (OTC) opiate abuse treatment. Ir I Psychol Med 2010; 27: 189-191.

43 Nielsen S, Bruno R, Murnion B, et al. Treating codeine dependence with buprenorphine: dose requirements and induction outcomes from a retrospective case series in New South Wales, Australia. Drug Alcohol Rev 2015; 35: 70-75.
44 Ammit M. Over-the-counter codeine dependency: a case analysis of an inpatient nursing intervention. Aust Nurs Midwifery J 2016; 23: 28-31.

45 Bedi RS. Dependence on a common cough linctus. Indian J Chest Dis Allied Sci 1991; 33: 227-228.

46 Dutch MJ. Nurofen Plus misuse: an emerging cause of perforated gastric ulcer. Med J Aust 2008; 188: 56-57. https://www.mja.com.au/journal/2008/188/ 1/nurofen-plus-misuse-emerging-cause-perforatedgastric-ulcer

47 Dyer BT, Martin JL, Mitchell JL, et al. Hypokalaemia in ibuprofen and codeine phosphate abuse. Int I Clin Pract 2004; 58: 1061-1062.

48 Eng EL, Lachenmeyer J. Codeine self-medication in a headache patient. Headache 1996; 36: 452-455.

49 Evans C, Chalmers-Watson TA, Gearry RB. Combination NSAID-codeine preparations and gastrointestinal toxicity. N Z Med J 2010; 123: 92-93.

50 Faierman $D$, Jacobs $S$. Liver injury from elixir of terpin hydrate with codeine. Mt Sinai J Med 1973. 40: 56-59.

51 Gruber CM, Nelson GM. Codeine addiction. Ann Intern Med 1948; 29: 151-153.

52 Hard B. Management of opioid painkiller dependence in primary care: ongoing recovery with buprenorphine/ naloxone. BMJ Case Rep 2014; doi: 10.1136/bcr2014-207308.

53 Karamatic R, Croese J, Roche E. Serious morbidity associated with misuse of over-the-counter codeine-ibuprofen analgesics. Med J Aust 2011; 195: 516. https://www.mja. com.au/journal/2011/195/9/serious-morbidityassociated-misuse-over-counter-codeine-ibuprofenanalgesics

54 Kean J. Illicit and over-the-counter codeine dependence after acute back pain-successful treatment and ongoing recovery after buprenorphine/ naloxone taper. Heroin Addict Relat Clin Probl 2016; 18: 21-24.

55 Lake IAE. Screening and behavioral management: medication overuse headache - the complex case. Headache 2008; 48: 26-31.

56 Lake $\mathrm{H}$. Ibuprofen belly: a case of small bowel stricture due to non-steroidal anti-inflammatory drug abuse in the setting of codeine dependence. Aust N Z J Psychiatry 2013; 47: 1210-1211.

57 Marr E, Hill D. Optimising service provision for prescribed opioid analgesic dependence. Heroin Addict Relat Clin Probl 2015; 17: 13-18.

$58 \mathrm{Ng} J \mathrm{~J}$, Morgan DJR, Loh NKM, et al. Life-threatening hypokalaemia associated with ibuprofen-induced renal tubular acidosis. Med J Aust 2011; 194: 313-316. https://www.mja.com. au/journal/2011/194/6/life-threateninghypokalaemia-associated-ibuprofen-inducedrenal-tubular

59 Page CB, Wilson PA, Foy A, et al. Life-threatening hypokalaemia associated with ibuprofen-induced renal tubular acidosis. Med J Aust 2011; 194: 613-614. https://www.mja.com.au/journal/2011/194/11/lifethreatening-hypokalaemia-associated-ibuprofeninduced-renal-tubular

60 Robinson GM, Robinson S, McCarthy P, Cameron C Misuse of over-the-counter codeine-containing analgesics: dependence and other adverse effects. N Z Med J 2010; 123: 59-64.

61 Roussin A, Bouyssi A, Pouche L, et al. Misuse and dependence on non-prescription codeine analgesics or sedative $\mathrm{Hl}$ antihistamines by adults: a crosssectional investigation in France. PLoS One 2013; 8: e76499.

62 Senjo M. Obsessive-compulsive disorder in people that abuse codeine. Acta Psychiatr Scand 1989; 79: 619-620. 
63 Vaughan JV, Fleischl P, Nathan M, Taylor RC. Chronic renal disease and analgesic abuse. N Z Med J 1967; 66 : 794-797.

64 Wylie AS, Fraser AA. Hazards of codeine plus paracetamol compounds. Br J Gen Pract 1994; 44: 376.

65 Van Hout MC, Delargy I, Ryan G, et al. Dependence on over the counter (OTC) codeine containing analgesics: treatment and recovery with buprenorphine naloxone. Int J Ment Health Addict 2016; 14: 873-883.

66 Romach MK, Otton SV, Somer G, et al. Cytochrome P450 2D6 and treatment of codeine dependence. $J$ Clin Psychopharmacol 2000; 20: 43-45.

67 Nilsen HK, Stiles TC, Landro Nl, et al. Patients with problematic opioid use can be weaned from codeine without pain escalation. Acta Anaesthesiol Scand 2010; 54: $571-579$

68 Fernandes LC, Kilicarslan T, Kaplan HL, et al. Treatment of codeine dependence with inhibitors of cytochrome P450 2D6. J Clin Psychopharmacol 2002; 22: 326-329.

69 Norman IJ, Bergin M, Parry CD, Van Hout MC. Best practices and innovations for managing codeine misuse and dependence. J Pharm Pharm Sci 2016; 19: 367-381.
70 Nielsen S, Larance B, Degenhardt L, et al. Opioid agonist treatment for pharmaceutical opioid dependent people. Cochrane Database Syst Rev 2016; (5): CDOו1ו17.

71 Mattick RP, Breen C, Kimber J, Davoli M. Buprenorphine maintenance versus placebo or methadone maintenance for opioid dependence. Cochrane Database Syst Rev 2014; (2): CD002207.

72 Gowing L, Ali R, Dunlop A, et al. National guidelines for medication-assisted treatment of opioid dependence. Canberra: National Drug Strategy, 2014. http://www. nationaldrugstrategy.gov.au/internet/drugstrategy/ Publishing.nsf/content/AD14DA97D8EE00E8CA257CD1 001E0E5D/\$File/National_Guidelines_2014.pdf (viewed Dec 2017).

73 Amato L, Minozzi S, Davoli M, Vecchi S. Psychosocial and pharmacological treatments versus pharmacological treatments for opioid detoxification. Cochrane Database Syst Rev 2011; (9): CD005031.

74 Amato L, Minozzi S, Davoli M, Vecchi S. Psychosocial combined with agonist maintenance treatments versus agonist maintenance treatments alone for treatment of opioid dependence. Cochrane Database Syst Rev 2011; (10): CD004147.
75 Quello SB, Brady KT, Sonne SC. Mood disorders and substance use disorder: a complex comorbidity. Sci Pract Perspect 2005; 3: 13-21.

76 Evers S, Jensen R. Treatment of medication overuse headache: guideline of the EFNS headache panel. Eur J Neurol 2011; 18: 1115-1121.

77 Chiang C, Schwedt T, Wang S, Dodick D. Treatment of medication-overuse headache: a systematic review. Cephalalgia 2016; 36: 371-386.

78 Tassorelli C, Jensen R, Allena M, et al. A consensus protocol for the management of medication-overuse headache: evaluation in a multicentric, multinational study. Cephalalgia 2014; 34: 645-655.

79 Katsarava Z, Fritsche G, Muessig M, et al. Clinical features of withdrawal headache following overuse of triptans and other headache drugs. Neurology 2001; 57: 1694-1698.

80 Dodick DW, Silberstein SD. How clinicians can detect, prevent and treat medication overuse headache. Cephalalgia 2008; 28: 1207-1217.

81 Katsarava Z, Muessig M, Dzagnidze A, et al. Medication overuse headache: rates and predictors for relapse in a 4-year prospective study. Cephalalgia 2005; 25: 12-15. 